ADLFI. Archéologie de la France -

INFORMATIONS Informations

une revue Gallia

Languedoc-Roussillon | 1997

\title{
Clermont-l'Hérault - L'îlot d'Enoz
}

\section{Astrid Huser}

URL : http://journals.openedition.org/adlfi/11660

ISSN : 2114-0502

Éditeur

Ministère de la culture

Référence électronique

Astrid Huser, «Clermont-l'Hérault - L'îlot d'Enoz », ADLFI. Archéologie de la France - Informations [En ligne], Languedoc-Roussillon, mis en ligne le 01 mars 2004, consulté le 25 avril 2019. URL : http:// journals.openedition.org/adlfi/11660

Ce document a été généré automatiquement le 25 avril 2019

(c) Ministère de la Culture et de la Communication, CNRS 


\title{
Clermont-l'Hérault - L'îlot d'Enoz
}

\author{
Astrid Huser
}

Date de l'opération : 1997 (EV) ; 1997 - 1998 (SU)

Inventeur(s) : Huser Astrid

1 Un château fort dressé sur le sommet d'une éminence, une première enceinte formant une vaste cour basse sous le château, l'agglomération castrale qui se développe sur les pentes et déborde en faubourgs à partir du $\mathrm{XIV}^{\mathrm{e}} \mathrm{s}$. rendent compte de la physionomie médiévale de Clermont-l'Hérault.

Des lacunes importantes restent à combler sur l'histoire de cette seigneurie laïque, la plus importante du diocèse de Lodève, à commencer par son origine (la première mention connue du castrum est tardive, 1158 et celle d'un Guilhem, baron de Clermont n'est pas antérieure à 1130).

3 C'est dans son agglomération castrale qu'une première étude de l'architecture civile a pu être conduite en avril 1997 dans le cadre de la démolition partielle d'un îlot, l'îlot d'Enoz, rendu vétuste par manque d'entretien.

4 L'observation cadastrale et aérienne du castrum met en évidence un maillage de voies préexistentes qui en contraint le développement urbain et dont l'îlot d'Enoz porte l'empreinte sur l'une de ses faces. Ce tracé, en forme d'Y part de l'église Saint-Paul, située extra-muros, enserre la place du Radical et remonte en se ramifiant jusqu'au portail neuf et les îlots qui ceinturent le bas de l'enceinte castrale (Fig. $n^{\circ} 1$ : Plan général.).

5 Cette configuration peut matérialiser d'une part l'existence d'un habitat primitif se dotant d'un lieu de culte, Saint-Paul et précédant l'arrivée aristocratique; d'autre part, elle suggère un premier noyau s'agglomérant au château avant de se développer entre les deux pôles que sont le château et l'église Saint-Paul et de recevoir une forme définitive par d'imposantes fortifications flanquées de tours dont la chronologie reste à établir. On sait qu'au XIV $\mathrm{X}^{\mathrm{e}}$., Saint-Paul est intégré au système défensif de la ville comme avantposte puissant. 
6 Sur le site, quelques éléments épars, de facture médiévale, ne permettent pas de caler chronologiquement la mise en place de l'îlot d'Enoz ni son rapport avec l'installation du rempart qui l'enclôt.

7 Les premiers vestiges en place se manifestent sous la forme d'une série d'arcs : trois arcs en tiers-point et un quatrième en plein-cintre reposent deux à deux sur un pilier central et sont surmontés d'un plein-de-travée. Interrompus de part et d'autre, cet ensemble, qui est datable des XIII ${ }^{e}$ s. - XIV ${ }^{e}$ s., ne révèle pas sa fonction : simple mitoyenneté ou arcade marchande?

Bridé par un parcellaire en place, la configuration de la ville à partir de cette époque est encore bien lisible : une enceinte flanquée de tours rondes et de portes enserre un habitat particulièrement dense dont l'encombrement se traduit en surcroîts, avances sur rue et porches et appelle les premières réglementations (transaction entre Bérenger, seigneur de Clermont et les syndics; cette transaction établit une existence communale et reconnaît que la ville forme une université, un corps, une communauté). Certaines maisons, composées de hautes et vastes pièces, sont décorées jusqu'au deuxième étage de peintures murales. Les vestiges d'un ensemble pictural ont pu être prélevés : œuvre d'un peintre local stylistiquement peu probable, cette peinture présente des éléments reconnaissables qui incitent à la dater de la fin $\mathrm{XIV}^{\mathrm{e}} \mathrm{s}$. ou au début $\mathrm{XV}^{\mathrm{e}}$ s. Plusieurs motifs sont repérables : un faux appareil polychrome au haut duquel surgit une tête hirsute animée de deux grands yeux surlignés au trait noir ainsi qu'un bouc; les bras remontés au niveau du visage suggèrent un étirement du sourire en grimace à l'aide des doigts! Un autre panneau présente à la verticale un signe en rouge pouvant représenter une lettre cabalistique ainsi qu'un motif rappelant l'empreinte de pattes d'oiseaux; en dessous, un homme de profil, muni d'un chapeau mou et allongé, délivre, par sa bouche entr'ouverte, une langue de feu. Une sorte de grille au trait noir complète cet ensemble.

Un scénario d'installation de l'îlot commence à se dessiner : le recours au corps double et au pan de bois semble commun et s'étendre à l'ensemble de la vieille ville.

Le corps double est une réponse au laniérage profond du parcellaire, aux nécessités d'éclairage et à la faiblesse des assemblages dans le Midi.

11 Le pan de bois, de type archaïque, voit sablières et poutres se répartir horizontalement les charges des étages, leurs bouts de poutre permettant la mise en place d'un surplomb; un avant-toit protège la façade en plâtre et chaux et favorise la mise en place d'un décor.

Les éléments perçus nous donne des indications sur l'espace intérieur du logis : la salle est le pôle autour duquel s'organisent les chambres ; la communication entre les étages doit se faire par un escalier droit ou à vis.

13 Un plafond à la française couvre chaque pièce ; celles-ci sont éclairées par des fenêtres à croisées et chauffées par une cheminée centrale; un placard muni d'un arc en pleincintre trouve sa place dans l'épaisseur du mur et un système d'évacuation des eaux en pierre relié à une fontaine à l'étage permet d'entrevoir certaines mesures de confort et d'hygiène ; un puits et une aire de stockage (cuves en grès monolithes) peuvent occuper le rez-de-chaussée...

14 La fin du XVI $\mathrm{e}_{\mathrm{e}}$ s. s'illustre par des façades en gypserie : grandes baies à croisées, hauteur des pièces soulignée par le jeu des bandeaux et des appuis, richesse du décor foisonnant selon un répertoire maniériste. La décoration agrémente également l'intérieur des maisons pour souligner clefs pendantes et rampes pleines d'escalier; les cheminées et fontaines font l'objet d'un soin particulier. La maison, sise 11, rue Fontaine-de-la-Ville, se 
distingue par sa façade sur rue en pan de bois avec surplomb tout à fait remarquable par son décor maniériste en gypserie, unique vestige de cette technique de décoration connu dans la région : deux grandes baies à croisées rassemblent pilastres à grandes palmettes et frises (l'une d'elles, supportée par les pilastres, est ornée de rinceaux et de guirlandes à fleurs articulées autour d'une tête d'angelot et de deux têtes de faunes latérales, tandis que l'autre souligne l'appui des fenêtres). Non protégée, cette façade vient d'être détruite. Calqué sur le modèle urbain, un exemple de vaste demeure « bourgeoise » s'organisant en un logis principal prolongé par deux corps de part et d'autre d'une cour et s'élevant sur trois étages nous est donné dans l'îlot. La façade présente un portique de l'époque classique auquel s'apparentent également les rampes à balustre de l'escalier intérieur et la porte à bossage accédant au premier étage ; cet étage fait l'objet d'un soin particulier et la décoration monumentale orne cheminées et plafonds moulurés. La maison est cependant plus ancienne si l'on en juge les bandeaux de façade sur rue et sur cour associées à des fenêtres initialement à croisées, ensuite transformées.

Le XVIII ${ }^{e}$ s. affiche une volonté de modernité avec la transformation des fenêtres à croisées en fenêtres couvertes d'un arc segmentaire et la mise en place de génoises; les cloisonnements, marquant remembrement et évolution fonctionnelle des pièces, impriment, dans la structure même du bâti, la lente mutation du mode de vie traditionnel (passage d'une occupation unifamiliale à des espaces privatifs, etc.).

17 Il semble également que la ville tente de faire peau neuve et aère le cœur de ville : en vue de l'alignement des rues, certaines maisons sont tronquées de leur surplomb initial (la façade en pan de bois n'étant pas porteuse, le problème de sa reprise complète ne se pose pas).

18 Les ferronneries ajourent les rampes d'escalier et l'ornementation en stuc gagne les intérieurs : pièces entièrement jalonnées de panneaux décoratifs caractéristiques du style Louis XVI témoignant, une fois encore, d'un souci de mise au goût du jour...

19 (Amado, Claudie. 1991-1992.), (Bourin-Derruau, Monique. 1987.), (Barral, Paul. 1918.), (Combarnous, Gaston. 1960.), (Schneider, Laurent. 1989.), (Sournia, Bernard ; Vayssettes, Jean-Louis. 1991.)

\section{BIBLIOGRAPHIE}

Amado, Claudie. 1991-1992 : « Aux origines des Guilhem de Montperllier, questions généalogiques et retour à l'historiographie ", Études sur l'Hérault, 7-8, p. 89-109.

Bourin-Derruau, Monique. 1987 : Villages médiévaux en Bas-Languedoc : genèse d'une sociabilité ( $X^{e}$ $X V I^{e}$ s., Paris, L'Harmattan, 2 vol., 338 p., 470 p.

Barral, Paul. 1918 : Considérations sur le régime municipal de Clermont en Lodévois aux WIII et XIV ${ }^{e}$ s., Émile Appolio, (diocèse civil de Lodève).

Combarnous, Gaston. 1960 : « Le développement de Clermont », Annales du Midi. 
Schneider, Laurent. 1989 : Lodevois et du Biterrois Nord-Oriental ( $V^{e}$-XIII ${ }^{e}$ siècles) : essai d'archéologie extensive, sous la dir. de Helas Jean-Claude et Sigal André, mémoire. de maîtrise d'Histoire, Montpellier, université Paul-Valéry, S.l., s.n., 396 p.

Sournia, BernardVayssettes, Jean-Louis. 1991 : Montpellier : la demeure médiévale, Paris, Imprimerie nationale, 256 p, coll. Études du patrimoine, 1.

\section{ANNEXES}

Fig. $\mathrm{n}^{\circ} 1$ : Plan général.

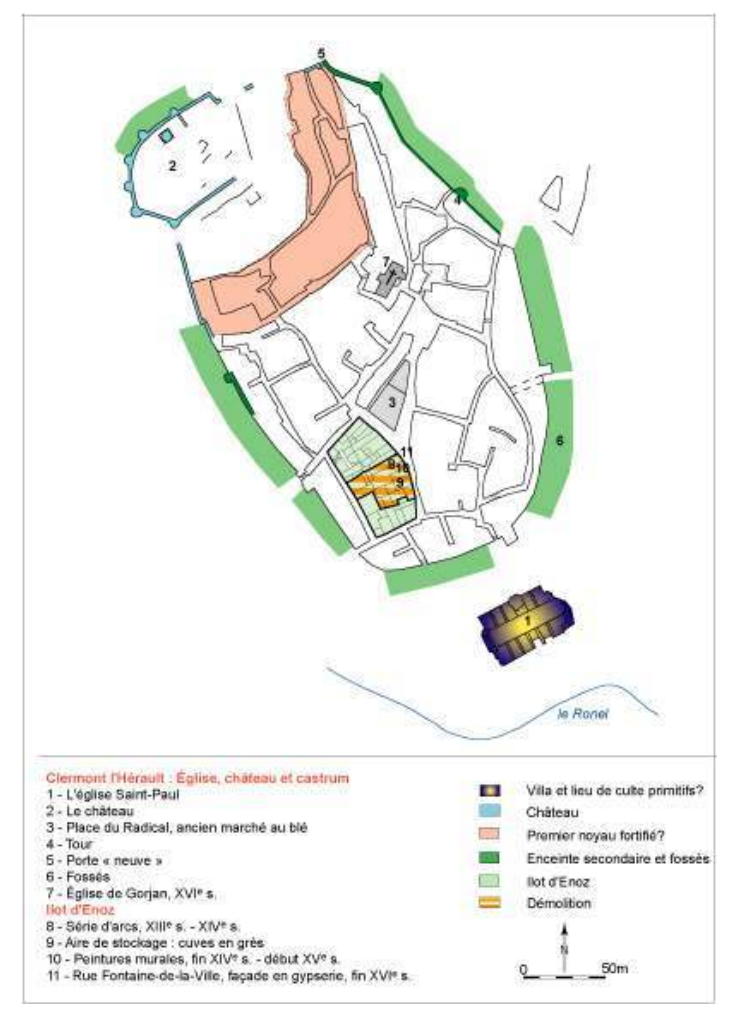

Auteur(s) : Huser, Astrid. Crédits : ADLFI (2004)

INDEX

Index chronologique : Moyen Âge*, Temps Modernes

Index géographique : Languedoc-Roussillon, Hérault (34), Clermont-l'Hérault operation Fouille d'évaluation (EV), Sauvetage urgent (SU) 